\title{
Collision-Balancing Frequency Hopping in Single-Hop Mobile Ad Hoc Networks
}

\author{
Ralph Tanbourgi*, Xevi Pujol i Molist ${ }^{\dagger}$, and Friedrich K. Jondral* \\ ${ }^{*}$ Communications Engineering Lab, Karlsruhe Institute of Technology, Germany \\ $\dagger$ Technical University of Catalonia (UPC), Spain \\ Email: \{ralph.tanbourgi, friedrich.jondral\}@kit.edu, xevi.pujol.molist@gmail.com
}

\begin{abstract}
We consider a single-hop frequency hopping (FH) mobile ad hoc network (MANET). To increase throughput orthogonal FH (O-FH) with hop-synchronous hopping can be used, thereby allowing collision-free transmissions under certain circumstances. However, as the MANET grows, hopping sequences must be re-used. As a result, nodes sharing the same sequence will experience deterministic collisions while others having a unique sequence will still communicate collision-free. This results in an unfair interference situation and makes performance guarantees difficult to meet for all nodes simultaneously. In this paper, we propose a collision-balancing FH (CB-FH) scheme to overcome this drawback. The CB scheme builds on O-FH and adapts the sequence set to balance interference among the nodes. We analytically study the characteristics of the adaptive sequences and analyze the resulting interference situation at the nodes. The performance is compared to uncoordinated FH (U-FH) and O-FH. It is found that CB-FH solves the problem of unfairness present in O-FH. Compared to U-FH, CB-FH yields considerable improvements in a wide range. Practical issues are discussed.

Index Terms-Adaptive frequency hopping, MANET, fairness.
\end{abstract}

\section{INTRODUCTION}

Mobile ad hoc networks (MANETs) have gained a lot of interest in the wireless community since the plurality of possible applications has been discovered. Driven by steady advances in hardware development that have led to small low-cost devices, MANETs have now become a promising approach to current and future challenges.

A MANET has to be adaptive and self-organizing in order to provide the desired services. In contrast to static (ad hoc) networks, sophisticated algorithms yielding optimal solutions are not applicable for MANETs in general, due to the fact that the network state inherently varies (sometimes unpredictably) on the small-scale resulting from node mobility [1]. The problem of dynamic topology, being one of the major challenges in MANETs, has to be tackled using a scalable management service [2]. Here, one has to trade-off optimality vs. control overhead, often precluding adaptation to the small-scale network dynamics. For certain (non-critical) management tasks, adaptation on a larger time scale is hence preferred, thereby adapting to the slowly-varying network dynamics.

Besides, medium access control is of eminent importance in MANETs, since self-interference is the performance bottleneck in most cases. Here, it is found that interference avoidance using FH is a promising strategy for MANETs [3].

FH based techniques can be roughly divided into two categories: Uncoordinated FH (U-FH) and (coordinated) orthogonal FH (O-FH). U-FH does not require the hopping sequences of concurrent nodes to be aligned in time which avoids sequence synchronization. However, it is well-known that letting nodes hop in an uncoordinated way leads to strong multiple access interference and inefficient spectrum usage. On the other hand, O-FH overcomes these problems by forcing the nodes to hop in a coordinated way. By appropriate alignment of the sequences in time, orthogonal hopping without collisions is possible when sufficient channels are available. However, as the network grows, some sequences have to be re-used, thereby removing the orthogonality property of OFH. Moreover, those nodes sharing the same sequence will experience deterministic collisions with one another, resulting in a strong temporal correlation of the interference. In contrast, nodes equipped with a unique sequence will not experience any interference. This fact causes the problem of unfairness in $\mathrm{O}-\mathrm{FH}$ which in turn complicates the satisfaction of quality of service requirements for all users simultaneously - a problem that represents another challenge in MANETs [4].

To overcome the problem of unfairness, we propose a collision-balancing (CB) FH scheme, a network management mechanism that builds on O-FH. The key idea is to adapt the hopping sequence set to the long-term network state by inserting some non-orthogonalities in an appropriate way such that the resulting sequences (i) minimize overall number of collisions and (ii) ensure an identical, and hence a fair interference situation in terms of collision probability for every node. Since the CB-FH scheme is supposed to operate on a large time-scale, it aims at satisfying (i) and (ii) in ergodic terms, i.e., as the spatial configuration of nodes varies sufficiently. The additional overhead resulting from exchanging control information and adapting the hopping sequence set remains low compared to conventional O-FH with nonadaptive sequences.

\section{A. Related work}

FH schemes designed for MANETs that take into account the network dynamics have been already studied in the literature. In [5], the authors propose a scheme to reduce the number of collisions in wireless personal area networks (WPANs) using orthogonal partitioning of the hopping set. This idea was further extended by [6], [7] by including an adaptation mechanism, which allows mutually interfering WPANs to 
appropriately choose their hopping sequence subset. While these approaches increase performance through orthogonal hopping, they do not modify the available set of hopping sequences, thereby limiting the obtainable gains. In [8], the authors propose a scheme that adapts the spacing of hopping channels depending on the actual network load, thereby modifying the number of available channels and hence, the hopping sequences. While the analysis demonstrates a considerable increase in spectral efficiency, practical implications, such as modification of filter bandwidths within the channel coherence time, seem not realistic and are not addressed in [8]. The idea of adapting the hopping sequence set to the network dynamics is proposed in [9]. Here, the problem of deterministic collisions when re-using orthogonal sequences is solved by a multilayer approach. Although this scheme achieves fairness among the nodes, it does not minimize overall number of collisions.

The rest of the paper is organized as follows: Section II explains the network model. In section III, the CB scheme is introduced and its characteristics are studied. An algorithm for generating the $\mathrm{CB}$ sequences is presented and practical issues are discussed. In section IV, the performance of CBFH is compared to U-FH and O-FH, followed by simulations validating the theoretical results. Section $\mathrm{V}$ gives a conclusion.

\section{NETWORK MODEL}

We assume a single-hop MANET consisting of $N$ nodes uniformly distributed on a disc $A \subset \mathbb{R}^{2}$ with radius $D$ according to a binomial point process $(\mathrm{BPP}) \Phi^{(N)}$. The positions of the nodes are given by $X_{1}, \ldots, X_{N} \in \Phi^{(N)}$. The total bandwidth is divided into a set $\mathcal{M}:=\left\{m_{1}, \ldots, m_{M}\right\}$ of $M$ orthogonal channels. ${ }^{1}$ Slow FH with slotted medium access is used, where "slow" means that hopping is performed on the order of a fixed packet duration. Every node $X_{i}$ has a hopping sequence $s_{i} \in \mathcal{M}^{L}$, where $L$ is the sequence length. If a node $X_{i}$ wants to transmit a message to a node $X_{j}$ in time slot $k$, it tunes to the channel corresponding to the current element $k$ of the hopping sequence $s_{j}$, i.e., $s_{j}(k)$.

We assume a path loss model, ${ }^{2}$ where the power path loss between two arbitrary points $x, y$ is $\frac{1}{\epsilon+\|x-y\|^{\alpha}}$, with path loss exponent $\alpha$. The parameter $\epsilon>0$ ensures boundedness of the path loss. Thermal noise is neglected (interference-limited regime) and transmission power is set to unity.

\section{THE CB-FH SCHEME}

\section{A. A motivating example}

To understand the basic idea of CB-FH assume the following situation: Let $N=5$ and $M=4$. In this case, no orthogonal sequence assignment is possible, and thus collisions must occur. But can we find a set of $N=5$ sequences such that these collisions affect all nodes in a balanced way, i.e., ensuring fairness among the nodes? Clearly, there should be $5-4=1$ total collisions in every hop at most in order

\footnotetext{
${ }^{1}$ With an additional common control channel. We assume that all nodes can exchange control information with each other via the control channel.

${ }^{2}$ Fading is not considered since it does not offer additional insights regarding the $\mathrm{CB}-\mathrm{FH}$ scheme.
}

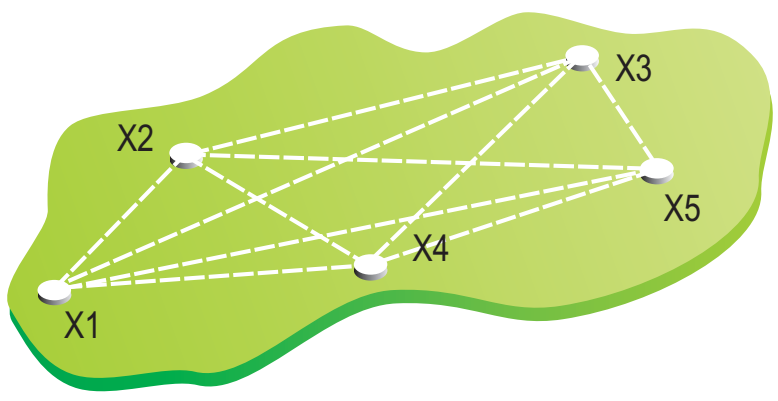

Fig. 1. Example of a single-hop MANET.

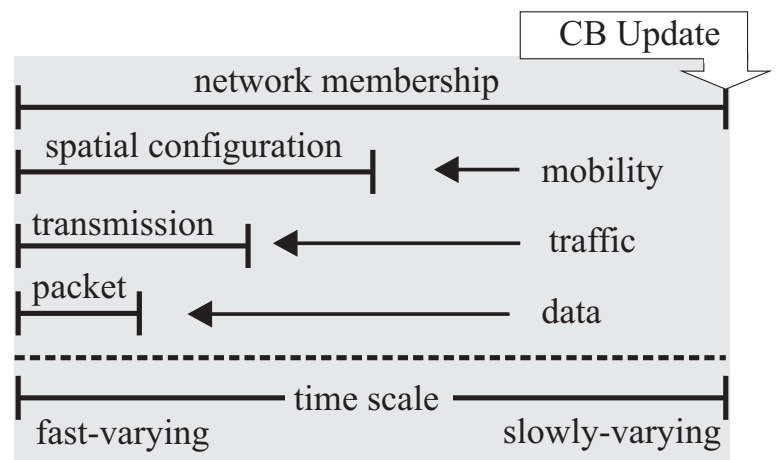

Fig. 2. Dynamics of a MANET. CB update frequency for information and sequence dissemination is on the order of network membership variation rate.

to minimize interference on the one hand, and to exploit the available bandwidth on the other hand. To provide fairness, the occurring collisions should not be experienced by only some of the 5 nodes as in $\mathrm{O}-\mathrm{FH}$, but they should be experienced by all of them by the same amount. In our example, the total number of possible collisions is $\frac{1}{2} \times 5 \times 4=10$, which can be illustrated by a complete graph, see Fig. 1 . The solution is to equalize the occurring collisions in time by "distributing" all 10 possible collisions evenly among the five nodes. By appropriate design of the sequences following this paradigm, we can let every node experience the same number of collisions while minimizing overall number of collisions.

From this example it can already be seen that the required network state information needed for the CB-FH scheme is simply $N$, i.e., the network membership. Although a MANET is inherently mobile, $N$ can be assumed only slowly-varying, e.g., in the presence of group mobility [10]. Fig. 2 illustrates the relevant dynamics of a MANET as well as the time scale on which the CB-FH scheme performs the adaptation.

\section{B. Sequence characteristics}

We now derive the characteristics of the CB-FH sequences. Let $\mathcal{C}$ be the set of all possible collision pairs and let $C_{\mathrm{h}}$ be the number of collisions occurring in a randomly chosen hop.

Lemma 1. For the CB-FH scheme with $N$ nodes and $M$ channels to be fairness-optimal, the sequence set must have

$$
\mathcal{C}= \begin{cases}\emptyset, & N \leq M \\ \bigcup_{\substack{1 \leq i, j \leq N \\ i \neq j}}\left(X_{i}, X_{j}\right), & M<N \leq 2 M\end{cases}
$$


non-orthogonalities (collision pairs) with a total of $|\mathcal{C}|=$ $\frac{1}{2} N(N-1)$ collisions for $M<N \leq 2 N$. To be interferenceoptimal, the sequence set must be designed such that

$$
C_{\mathrm{h}}= \begin{cases}0, & N<M \\ N-M, & M \leq N \leq 2 M\end{cases}
$$

collisions occur in each hop.

Proof: For the case $N \leq M$, it is possible to have a collision-free situation. Therefore, orthogonality of the sequences is preserved as in the case of O-FH. For the case $M<N \leq 2 M$, collisions will occur in every hop unavoidably. To avoid excessive interference, all $M$ channels should be used in every hop, leaving $N-M$ collisions unsolved. To be fairness-optimal, we let all nodes experience collisions, thereby creating $|\mathcal{C}|=\frac{1}{2} N(N-1)$ non-orthogonalities.

Lemma 2. The sequence length $L$ is given by

$$
L= \begin{cases}M, & 1 \leq N \leq M \\ \frac{N(N-1)}{2(N-M)}, & M<N \leq 2 M .\end{cases}
$$

Proof: This Lemma follows directly from Lemma 1.

Note that if $L$ is non-integer, we can always take a multiple of $L$ to perfectly balance the collisions.

Theorem 1. Let $N$ and $M, 1 \leq N \leq 2 M$, be the number of nodes and the number of available channels, respectively. Then, the collision probability $p^{\mathrm{CB}}$ at an arbitrary node for the CB-FH scheme in a randomly chosen hop is given by

$p^{\mathrm{CB}}:=\mathbb{P}($ one collision $)= \begin{cases}0, & 1 \leq N \leq M \\ 2\left(1-\frac{M}{N}\right), & M<N \leq 2 M .\end{cases}$

Proof: For $1 \leq N \leq M$, we have a collision-free situation, and hence $p^{\mathrm{CB}}=0$. For $M<N \leq 2 M$, there will be $C_{\mathrm{h}}$ collisions in each hop, affecting $2 C_{\mathrm{h}}$ nodes. By design, all nodes will experience the same number of collisions within one sequence period. Moreover, no node will experience collisions with more than one node. By combining these observations, we conclude that, in a randomly chosen hop, each node experience a collision with probability $\frac{2(N-M)}{N}$.

The case $N>2 M$ is not considered here since the resulting collision probability would be $p^{\mathrm{CB}}=1$ by construction.

\section{Sequence set generation and practical issues}

Next, we propose an algorithm for generating the $\mathrm{CB}$ sequence set. The very simple though efficient algorithm is shown at the top of the page.

The assignment of channels should be done in an "appropriate" way as indicated in the algorithm. This means, the channels should be chosen such that the resulting sequences have pseudo-random character. To further ensure an even distribution of collisions over the whole sequence length, a random permutation in time direction can be performed. Note that the permutation must be done to all sequences in the same way in order to maintain the desired non-orthogonalities.

The sequence set adaptation mechanism requires knowledge about the presence of nodes in the single-hop network. This

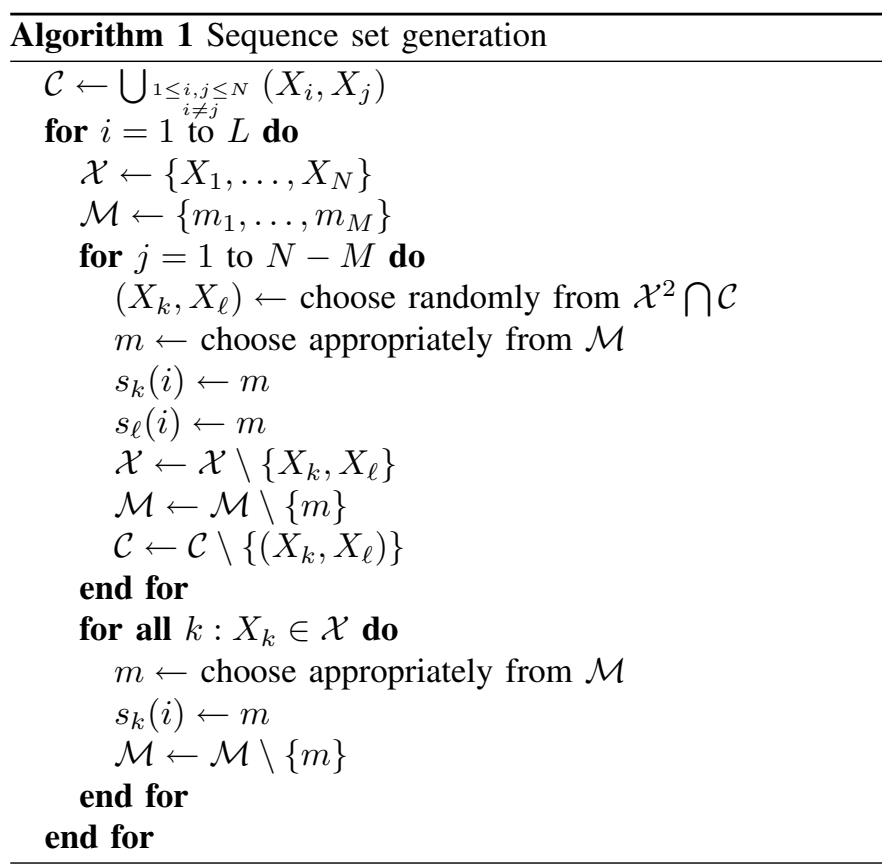

information has to be known at the node that is elected to perform the modification of the sequence set. This procedure is part of the self-organization task and can be efficiently accomplished in single-hop ad hoc networks, cf. [11]. For example, a leader node monitors the total number of nodes in the network and, in case this number changes, triggers the remaining nodes to change/adapt their hopping sequence. The triggering action may moreover carry additional content, e.g., hop synchronization information, etc.

\section{PERFormance ANAlysis}

\section{A. Hit probability}

An often used metric for studying the performance of $\mathrm{FH}$ sequences is the so-called hit probability [12], which is, from the viewpoint of a node $X_{i}$, the probability that at least one node $X_{j}$ uses the same channel at the same time (same slot $k$ ), i.e., $s_{i}(k)=s_{j}(k)$. The hit probability is given by

$$
p_{\mathrm{h}}:=\mathbb{P}(\text { at least one collision }) .
$$

The hit probability takes into account neither the relative distances between the nodes nor the physical channel. However, in single-hop ad hoc networks, where each node can interfere with all others, the hit probability is an appropriate metric. Note that $p^{\mathrm{CB}}$ in (4) is equal to the hit probability $p_{\mathrm{h}}$ since a node can experience at most one collision per hop by construction.

1) Uncoordinated $\mathrm{FH}$ : For U-FH, there is no sequence synchronization that ensures orthogonality between the nodes. As a consequence, nodes may possibly experience one or more collisions in one hop. The hit probability for U-FH is

$$
p^{\mathrm{U}}:=\mathbb{P}(\text { at least one collision })=1-\left(1-\frac{1}{M}\right)^{N-1} \text {. }
$$


2) Orthogonal FH: To increase system throughput, O-FH can be employed. In the case where $N \leq M$, nodes do not experience any collisions. However, when $N>M$, some hopping sequence have to be re-used. Consequently, some nodes will still be able to communicate collision-free, while those nodes sharing the same sequences will experience deterministic collisions. The hit probability for O-FH is therefore given by

$$
\begin{aligned}
p^{\mathrm{O}} & :=\mathbb{P}(\text { at least one collision }) \\
& =\mathbb{P}(\text { one collision })=\left\{\begin{array}{l}
1, \text { if re-used sequence } \\
0, \text { if unique sequence. }
\end{array}\right.
\end{aligned}
$$

3) Comparison hit probability: Now, we compare the hit probabilities $p_{\mathrm{h}}$ of the three schemes, i.e., we consider (4), (6) and (7). The hit probabilities are shown in Fig. 3. Comparing CB-FH and U-FH, we see that CB-FH has lower hit probability in a wide range. While in case of U-FH, collisions can occur even when $N \leq M$, CB-FH creates no collisions. Inserting non-orthogonalities as done for $\mathrm{CB}-\mathrm{FH}$ remains beneficial compared to U-FH until the ratio $N / M$ exceeds a certain value. This value can be computed using the approximation $1-\left(1-\frac{1}{M}\right)^{N-1} \approx 1-e^{-\frac{N}{M}}$, yielding

$$
2\left(1-\frac{M}{N}\right) \stackrel{!}{=} 1-e^{-\frac{N}{M}} \Rightarrow \frac{N}{M} \approx 1.68 .
$$

When the ratio $N / M$ is greater 1.68 , U-FH becomes superior to CB-FH. Both schemes are equally fair since the hit probability is the same for all nodes.

Comparing CB-FH with O-FH, we observe that both schemes have hit probability equal to zero when $N \leq M$. This is due to the fact that in this regime, a collisionfree assignment is possible. However, as we proceed to the regime $M<N \leq 2 M$, some sequences are re-used in the O-FH scheme, thereby creating deterministic collisions between certain nodes $\left(p^{\mathrm{O}}=1\right)$, while others still can transmit collision-free $\left(p^{\mathrm{O}}=0\right)$. Therefore, we conclude that $\mathrm{O}-\mathrm{FH}$ is inferior to both CB-FH and U-FH in terms on fairness.

\section{B. Sum interference}

A metric that takes into account physical aspects of the communication channel is given by the sum interference. The channel model introduced in Section II will be adopted. To analyze the sum interference, we have to specify at which location it is measured, thereby implicitly conditioning $\Phi^{(N)}$ on having a node at this location. This node will be called the probe node, as it will help us to measure the sum interference. Without loss of generality, we assume that $X_{1} \equiv x_{1}$ is our probe node. Before continuing with the analysis, we first have to check how this conditioning affects the distribution of $\Phi^{(N)}$.

Lemma 3. Conditioned on $X_{1} \equiv x_{1}$, the positions of the remaining nodes $X_{2}, \ldots, X_{N}$ follow a BPP $\Phi^{(N-1)}$.

Proof: From Slivnyak's Theorem [13] it follows that $\mathbb{P}\left(\Phi^{(N)} \backslash\left\{x_{1}\right\} \in \cdot \mid x_{1} \in A\right)$ is equal to $\mathbb{P}\left(\Phi^{(N-1)} \in \cdot\right)$, since the node positions $X_{1}, \ldots, X_{N}$ are independent.

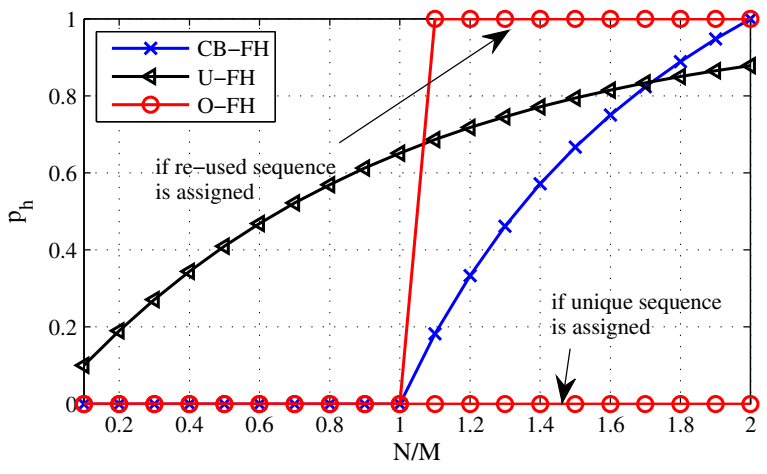

Fig. 3. Hit probabilities $p_{\mathrm{h}}$ for the three schemes

Furthermore, a parameter $\lambda \in[0,1]$ denoting the fraction of active transmitters, is used to study how the network load affects the CB-FH performance. For $\lambda=1 / N$, there will be only one node transmitting and all $N-1$ other nodes receiving, e.g., broadcast transmission, or inactive. In contrast, when $\lambda=$ 1 , all nodes are transmitting at the same time, e.g., full-duplex transmission. We assume that all nodes independently decide whether to transmit or not with probability $\lambda$.

Hence, the sum interference can be written as

$$
I\left(x_{1}\right):=\sum_{i=2}^{N} \frac{B_{i}}{\epsilon+\left\|X_{i}-x_{1}\right\|^{\alpha}},
$$

where

$$
B_{i}= \begin{cases}1, & \text { if } X_{i} \text { is } \mathrm{Tx} \text { and hits node } x_{1} \\ 0, & \text { otherwise. }\end{cases}
$$

We now compute the expected sum interference $\mathbb{E}\left[I\left(x_{1}\right)\right]$ :

Theorem 2. The expected sum interference at $x_{1} \in A \subset \mathbb{R}^{2}$ is given by

$$
\mathbb{E}\left[I\left(x_{1}\right)\right]=\frac{C_{1} \lambda}{\pi D^{2}} \int_{0}^{D} \int_{0}^{\pi} \frac{2 r \mathrm{~d} \theta \mathrm{d} r}{\epsilon+\left(r^{2}+\left\|x_{1}\right\|^{2}-2 r\left\|x_{i}\right\| \cos \theta\right)^{\frac{\alpha}{2}}}
$$

where

$$
C_{1}:= \begin{cases}\frac{N-1}{M}, & \text { for } \mathrm{U}-\mathrm{FH} \\ p^{\mathrm{O}}, & \text { for } \mathrm{O}-\mathrm{FH} \\ p^{\mathrm{CB}}, & \text { for } \mathrm{CB}-\mathrm{FH} .\end{cases}
$$

Proof: We write

$$
\begin{aligned}
& \mathbb{E} {\left[I\left(x_{1}\right)\right]=\sum_{i=2}^{N} \mathbb{E}\left[B_{i}\right] \mathbb{E}\left[\frac{1}{\epsilon+\left\|X_{i}-x_{1}\right\|^{\alpha}}\right] } \\
&= \sum_{i=2}^{N} \mathbb{E}\left[\mathbb{E}\left[B_{i} \mid X_{i} \text { is } \mathrm{Tx}\right]\right] \frac{1}{\pi D^{2}} \int_{0}^{D} \int_{0}^{\pi} \frac{2 r \mathrm{~d} \theta \mathrm{d} r}{\epsilon+\left\|(r, \theta)-x_{1}\right\|^{\alpha}} \\
&= \underbrace{(N-1) \mathbb{P}\left(\text { node hits } x_{1}\right)}_{0} \frac{\lambda}{\pi D^{2}} \\
& \times \int_{0}^{D} \int_{0}^{\pi} \frac{2 r \mathrm{~d} \theta \mathrm{d} r}{\epsilon+\left(r^{2}+\left\|x_{1}\right\|^{2}-2 r\left\|x_{i}\right\| \cos \theta\right)^{\frac{\alpha}{2}}}
\end{aligned}
$$


(13) follows from the independence between $B_{i}$ and the positions $X_{i}$. In (14), we have applied the law of the iterated expectation. Furthermore, we have performed a transformation to polar coordinates $\theta, r$, to evaluate the right-hand expectation of (13). We have used the fact that $X_{2}, \ldots, X_{N}$ are i.i.d. with density $f_{\Theta, R}(\theta, r)=\frac{1}{2 \pi} \frac{2 r}{D^{2}}$, to compute this expectation. In (15) we have further used the fact that $\mathbb{E}\left[\mathbb{E}\left[B_{i} \mid X_{i}\right.\right.$ is Tx $\left.]\right]$ breaks down to the product $\lambda \mathbb{P}\left(X_{i}\right.$ hits $\left.x_{1}\right)$, which is equal for all $N-1$ nodes.

Corollary 1. For $x_{1}=0$, the expected sum interference is given in closed-form by $\lambda \frac{C_{1}}{\epsilon}{ }_{2} F_{1}\left(\frac{2}{\alpha}, 1 ; 1+\frac{2}{\alpha} ; \frac{-D^{\alpha}}{\epsilon}\right)$, where ${ }_{2} F_{1}(a, b ; c ; d)$ is the hypergeometric function [14].

Let $S\left(x_{1}\right)$ be the desired received power at the receiver $x_{1}$. Further, we say that $x_{1}$ is in outage if the signal-tointerference ratio $\frac{S\left(x_{1}\right)}{I\left(x_{1}\right)}$ is below a threshold $\beta$. The next corollary demonstrates how (11) can be used to analyze the outage probability $(\mathrm{OP})$ in our single-hop MANET:

Corollary 2. The OP $q$ can be bounded as ${ }^{3}$

$$
\left.\begin{array}{r}
\lambda p^{\mathrm{CB}} C_{2} \\
\lambda p^{\mathrm{O}} C_{2} \\
1-e^{-\frac{\lambda}{M} C_{2}}
\end{array}\right\} \leq q\left(x_{1}\right) \leq \frac{\beta}{S\left(x_{1}\right)} \mathbb{E}\left[I\left(x_{1}\right)\right],
$$

where $C_{2}:=\mathbb{P}\left(b\left(x_{1},\left(\beta / S\left(x_{1}\right)\right)^{1 / \alpha}\right) \cap A \neq \emptyset\right)$ and $b(x, r)$ is a disc with radius $r$ centered at $x$.

Proof: The lower bound is constructed by considering the probability of a dominant interferer, which is a sufficient condition for outage, cf. [15]. For a transmitter to be dominant, it has to be located within the distance $\left(\beta / S\left(x_{1}\right)\right)^{1 / \alpha}$ to $x_{1}$, thereby directly creating outage. The upper bound follows from the Markov inequality $\mathbb{P}(|X| \geq x) \leq \frac{1}{x} \mathbb{E}[|X|]$.

Fig. 4 shows the OP $q$ (lower bound) vs. $N / M$ for different network loads $\lambda$. The upper bound is not shown because it is very loose (cf. [15]), while the lower bound is tight as indicated by the simulation results. It can be seen that CB$\mathrm{FH}$ is superior to $\mathrm{O}-\mathrm{FH}$ for $N / M>1$ : The $\mathrm{OP}$ for all users is considerable lower than the worst-case OP of OFH. For $N / M \rightarrow 1_{+}$, this gain tends to infinity, i.e., a large amount of unfairness is removed. Compared to U-FH, CBFH significantly reduces OP. The effect of different network loads is only marginal. However, recalling (16) and noting that $1-e^{-c} \leq c$, we see that the OP grows slower in $\lambda$ for $\mathrm{U}-\mathrm{FH}$ than for $\mathrm{CB}-\mathrm{FH}$ and $\mathrm{O}-\mathrm{FH}$.

\section{CONCLUSION}

We have proposed a collision-balancing frequency hopping (CB-FH) scheme for MANETs. The CB-FH scheme builds on orthogonal $\mathrm{FH}$ and aims at providing a fair interference situation among the nodes, thereby facilitating meeting quality of service requirements for all nodes simultaneously. The scheme adapts the hopping set to the number of nodes in the network, which despite node mobility can be assumed slowly-varying,

\footnotetext{
${ }^{3}$ Here, we have used the approximation $\left(1-\frac{x}{N}\right)^{N-1} \approx e^{-x}$ for U-FH.
}

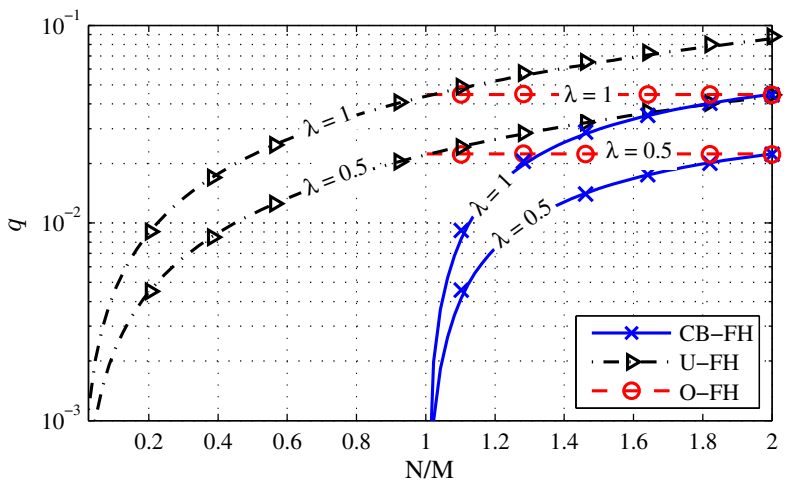

Fig. 4. OP $q$ vs. $N / M$. Solid lines represent lower bound from (16). Marks indicate the simulation results. The simulation parameters are: $D=100$, $x_{1}=(D / 2,0), \beta=2, S\left(x_{1}\right)=-50 \mathrm{~dB}, \epsilon=1, \alpha=4$.

e.g., in presence of group mobility. The resulting hopping sequence set (i) minimizes the total number of collisions and (ii) ensures an identical, and hence a fair interference situation at every node. After studying the characteristics of the adapted sequences, the performance of $\mathrm{CB}-\mathrm{FH}$ was analyzed and compared to O-FH and U-FH, the latter being fair but results in low spectrum efficiency. Simulation results confirm our analysis.

\section{REFERENCES}

[1] J. Hoebeke, I. Moerman, B. Dhoedt, and P. Demeester, "An overview of mobile ad hoc networks: Applications and challenges," Journal of the Communications Network, vol. 3, July 2007.

[2] D. P. G. Pravin Ghosekar, Girish Katkar, "Article:mobile ad hoc networking : Imperatives and challenges," IJCA Special Issue on MANETs, no. 3, pp. 153-158, 2010, published by Foundation of Computer Science.

[3] J. Andrews, S. Weber, and M. Haenggi, "Ad hoc networks: To spread or not to spread," IEEE Commun. Magazine, Dec. 2007.

[4] P. Goyal, V. Parmar, and R. Rishi, "Manet: Vulnerabilities, challenges, attacks, application," IJCEM, vol. 11, no. Jan., pp. 32-37, 2011.

[5] Z. Jiang, V. Leung, and V. Wong, "Reducing collisions between bluetooth piconets by orthogonal hop set partitioning," in Proc. of the RAWCON 2003, Aug. 2003, pp. $229-232$.

[6] P. Popovski, H. Yomo, and S. Aprili, "Frequency rolling: a cooperative frequency hopping for mutually interfering wpans," in Proc. of 5th ACM MobiHoc '04. New York, NY, USA: ACM, 2004, pp. 199-209.

[7] P. Popovski, H. Yomo, and R. Prasad, "Dynamic adaptive frequency hopping for mutually interfering wireless personal area networks," IEEE Trans. Mobile Computing, vol. 5, pp. 991-1003, 2006.

[8] K. Hamdi and O. Bamahdi, "A new adaptive frequency hopping technique," in IEEE Vehicular Technology Conference Fall (VTC), vol. 3 , Sep. 2004 , pp. 2083 - 2086 Vol. 3.

[9] J. Elsner, R. Tanbourgi, and F. K. Jondral, "Multiple access interference mitigation through multi-level locally orthogonal fh-cdma," in Military Communications Conference 2011, Nov. 2011.

[10] J.-C. Cano, P. Manzoni, and M. Sanchez, "Evaluating the impact of group mobility on the performance of mobile ad hoc networks," in IEEE Int. Conf. Commun. (ICC), vol. 7, June 2004, pp. 4039 - 4043 Vol.7.

[11] K. Nakano and S. Olariu, "Randomized initialization protocols for ad hoc networks," IEEE Trans. on Parallel and Distributed Systems, vol. 11, no. 7, pp. $749-759$, July 2000.

[12] E. Geraniotis and M. Pursley, "Error probabilities for slow-frequencyhopped spread-spectrum multiple-access communications over fading channels," IEEE Trans. Commun., vol. 30, no. 5, May 1982.

[13] A. Baddeley, "Spatial point processes and their applications," in Stochastic Geometry: Lectures given at the C.I.M.E. 2004, ser. Lecture Notes in Mathematics 1892. Springer Verlag, 2006, pp. 1-75.

[14] I. S. Gradshteyn and I. M. Ryzhik, Table of integrals, series, and products, 7th ed. Elsevier/Academic Press, Amsterdam, 2007.

[15] S. Weber, J. Andrews, and N. Jindal, "An overview of the transmission capacity of wireless networks," IEEE Trans. Commun., Dec. 2010. 